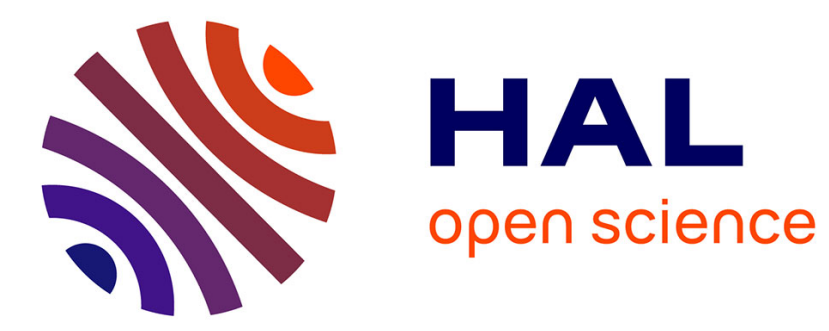

\title{
A generalization of Fan's matching theorem
}

Souhail Chebbi, Pascal Gourdel, Hakim Hammami

\section{To cite this version:}

Souhail Chebbi, Pascal Gourdel, Hakim Hammami. A generalization of Fan's matching theorem. 2008. hal-00756058v3

\section{HAL Id: hal-00756058 \\ https://shs.hal.science/hal-00756058v3}

Submitted on 6 Feb 2008

HAL is a multi-disciplinary open access archive for the deposit and dissemination of scientific research documents, whether they are published or not. The documents may come from teaching and research institutions in France or abroad, or from public or private research centers.
L'archive ouverte pluridisciplinaire HAL, est destinée au dépôt et à la diffusion de documents scientifiques de niveau recherche, publiés ou non, émanant des établissements d'enseignement et de recherche français ou étrangers, des laboratoires publics ou privés. 


\section{Centre d'Economie de la Sorbonne}

UMR 8174

A Generalization of Fan's Matching Theorem

Souhail CHEBBI

Pascal GOURDEL

Hakim HAMMAMI

(Version révisée)

2006.60
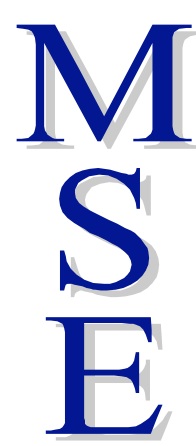

de

la

CENTRE NATIONAL

DE LARECHERCHE

SCENTIFIQUE 


\title{
A Generalization of Fan's matching Theorem
}

\author{
S. CHEBBI, P. GOURDEL and H. HAMMAMI
}

\begin{abstract}
We introduce a generalized coercivity type condition for set-valued maps defined on topological spaces endowed with a generalized convex structure and we extend the Fan's matching theorem.
\end{abstract}

The purpose of this note is to study a coercivity type condition for set-valued maps defined in L-spaces and to extend the non-compactness condition of Fan-KKM's matching theorem.

We recall the L-structure of convexity for topological spaces given in [BCFL]. This extension of usual convexity encompasses many others (H-convexity introduced by Horvath [H1, H2, H3], G-convexity introduced by Ding and Tan [DT] ...). The notion of KKM maps is easily extended to L-spaces. We then introduce the concept of L-coercing family for set-valued maps defined in Lspaces and give some examples. This coercivity type condition extends the one defined in Hausdorff topological vector spaces by Ben-El-Mechaiekh, Chebbi and Florenzano in [BCF]. The main result that we obtain is a Fan's type matching theorem concerning the intersection of KKM set-valued maps with quasi-compactly closed values and admitting a coercing family. A result on fixed point is then deduced. All these results extend classical results obtained in topological vector spaces by Fan in [F2] and Ding and Tan in [DT] as well as results obtained in H-spaces by Bardaro and Ceppitelli in [BC1] and [BC2] or in convex spaces in the sense of Lassonde in [L].

In what follows, the family of all nonempty finite subset of any set $X$ is denoted by $\langle X\rangle$. If $X$ is a vector space, the convex hull of a subset $A$ of $\mathrm{X}$ is denoted by $\operatorname{conv} A$. Since topological spaces in this paper are not supposed to be Hausdorff, following the terminology used in [B], a set is called quasi-compact if it satisfies the Finite Intersection Property while a Hausdorff quasicompact is called compact. If $n$ is any integer, $\Delta_{n}$ will denote the unit-simplex of $\mathbb{R}^{n}$ and for every $J \subset\{0,1, \ldots, n\}, \Delta_{J}$ denotes the face of $\Delta_{n}$ corresponding to $J$. Set-valued maps (or multifunctions) will be simply called maps and represented by capital letters $F, G, Q, S, \Gamma, \ldots$ Functions in the usual sense will be represented by small letters. If $X$ and $Y$ are two topological spaces, $\zeta(X, Y)$ denotes the set of all continuous functions from $X$ to $Y$.

Let $X$ be a topological space. An $L$-structure (also called $L$-convexity) on $X$ is given by a nonempty valued map $\Gamma:\langle X\rangle \rightarrow X$ such that for every $A=\left\{x_{0}, \ldots, x_{n}\right\} \in\langle X\rangle$, there exists a continuous function $f^{A}: \Delta_{n} \rightarrow \Gamma(A)$ such that for all $J \subset\{0, \ldots, n\}, f^{A}\left(\Delta_{J}\right) \subset \Gamma\left(\left\{x_{j}, j \in J\right\}\right)$.

2000 Mathematical Subject Classification: Primary 47H04, 47H10, 54H25.

Key words and phrases: L-structures, non-Hausdorff topology, KKM maps, L-coercing family, Fan-KKM theorem, fixed points.

Chebbi has been supported by College of Sciences-Research center project No (Math/2007/51).

The authors are grateful to the referee for her/his valuable comments. The authors thank J.-M. Bonnisseau for stimulating comments.

Corresponding author: Pascal GOURDEL, Paris School of Economics, University of Paris 1, CNRS, CES, Université de Paris 1 Panthéon-Sorbonne, 106 Boulevard de l'Hôpital, 75647 Paris cedex 13. 
Such a pair $(X, \Gamma)$ is called an $L$-space. A subset $C \subset X$ is said to be $L$-convex if for every $A \in\langle C\rangle$, $\Gamma(A) \subset C$. A subset $P \subset X$ is said to be $L$-quasi-compact if for every $A \in\langle X\rangle$, there is a quasicompact L-convex set $D$ such that $A \cup P \subset D$. Clearly, if $C$ is an L-convex subset of an L-space $(X, \Gamma)$, then the pair $\left(C, \Gamma_{\left.\right|_{\langle C\rangle}}\right)$ is an L-space.

It should be noticed that the L-convexity is different from the G-convexity defined by Park and Kim in $[\mathrm{PK}]$ which assume in addition the following condition:

$$
\text { For all } A, B \in\langle X\rangle, A \subset B \text { implies } \Gamma(A) \subset \Gamma(B) \text {. }
$$

The class of L-spaces contains topological vector spaces and their convex subsets as well as number of spaces with abstract topological convexity (examples of L-space are given in [BCFL], see also the thesis of Chebbi [C] for a survey on this literature). The notion of L-quasi-compactness generalizes the $\mathrm{H}$-compactness given in $[\mathrm{BC} 1]$ and the c-compactness defined in $[\mathrm{L}]$.

The notion of KKM maps is easily extended to L-spaces as follows:

Definition 1. Let $(X, \Gamma)$ be an L-space and $Z \subset X$ an arbitrary subset. $A$ map $F: Z \rightarrow X$ is called $K K M$ if and only if:

$$
\forall A \in\langle Z\rangle, \quad \Gamma(A) \subset \bigcup_{x \in A} F(x)
$$

We now introduce the notion of coercing family in L-spaces for a given map:

Definition 2. Let $Z$ be an arbitrary set in an $L$-space $(X, \Gamma), Y$ a topological space and $s \in \zeta(X, Y)$. A family $\left\{\left(C_{a}, K\right)\right\}_{a \in X}$ is said to be $L$-coercing for a map $F: Z \rightarrow Y$ with respect to $s$ if and only if:

(i) $K$ is a quasi-compact subset of $Y$;

(ii) For each $A \in\langle Z\rangle$, there exists a quasi-compact L-convex set $D^{A}$ in $X$ containing $A$ such that:

$$
x \in D^{A} \Rightarrow C_{x} \cap Z \subset D^{A} \cap Z ;
$$

(iii) $\left\{y \in Y: y \in \bigcup_{z \in s^{-1}(y)} \bigcap_{x \in C_{z} \cap Z} F(x)\right\} \subset K$.

Remark 1. It is easy to see that in the previous definition, condition (ii) implies that for all $x \in Z$, $C_{x}$ is an L-quasi-compact subset of $X$.

Definition 3. If $X$ is a topological space, a subset $B$ of $X$ is called quasi-compactly closed (open respectively) if for every quasi-compact set $K$ of $X, B \cap K$ is closed (open, respectively) in $K$.

Remark 2. Some authors define the notion of compactly-closed when for every compact set $K$ of $X, B \cap K$ is closed (open, respectively) in $K$. It is easy to check that if $A$ is quasi-compactly closed, it is compactly closed. 
Remark 3. Since condition (iii) can be rewritten as follows:

we can deduce that:

$$
\left\{z \in X: s(z) \in \bigcap_{x \in C_{z} \cap Z} F(x)\right\} \subset s^{-1}(K),
$$

(1) If $X$ is quasi-compact, then (iii) is automatically satisfied.

(2) If the family $\left\{\left(C_{a}, K\right)\right\}_{a \in X}$ is reduced to one element $\{(C, K)\}$ and the L-convexity is reduced to the usual convexity of a topological vector space, by putting s the identity function, condition (iii) of Definition 2 is reduced to the condition used in Theorem 4 of [F2].

(3) If $s(X)$ is contained in a quasi-compact subset of $Y$, then (iii) is automatically satisfied.

For any map $F: X \rightarrow Y$, let $F^{*}: Y \rightarrow X$ be the "dual" map of $F$ defined, for all $y \in Y$, by $F^{*}(y)=X \backslash F^{-1}(y)$, where $F^{-1}(y)=\{x \in X: y \in F(x)\}$.

Remark 4. By the previous remark and if we take $s$ the identity map, we can see that the sense of coercivity of Definition 2 comes from the fact that condition (iii) can be formulated for $F^{*}$, as the following condition:

$$
\forall y \in Y \backslash K, \quad F^{*}(y) \cap C_{y} \neq \emptyset
$$

which means that outside of the quasi-compact set $K$, we have some control on the values of $F^{*}$.

Let us now recall the coercivity in the sense of Ben-El-Mechaiekh, Chebbi and Florenzano in $[\mathrm{BCF}]$.

Definition 4. Consider a subset $X$ of an Hausdorff topological vector space and a topological space $Y$. Let I be nonempty, a family $\left\{\left(C_{i}, K_{i}\right)_{i \in I}\right\}$ of pairs of sets is said to be coercing for a map $F$ : $X \rightarrow Y$ if and only if:

(i) For each $i \in I, C_{i}$ is contained in a compact convex subset of $X$, and $K_{i}$ is a compact subset of $Y$;

(ii) For each $i, j \in I$, there exists $k \in I$ such that $C_{i} \cup C_{j} \subset C_{k}$;

(iii) For each $i \in I$, there exists $k \in I$ with $\cap_{x \in C_{k}} F(x) \subset K_{i}$.

In the following result, we prove that L-coercing families contain coercing families in the sense of Ben-El Mechaiekh, Chebbi and Florenzano.

Proposition 1. Let $X$ be an Hausdorff topological vector space and let $Y$ be a topological space. Let us assume that the map $F: X \rightarrow Y$ satisfy the coercivity condition of [BCF] (Definition 4). Then there exists some family $\left\{\left(\tilde{C}_{a}, K\right)\right\}_{a \in X}$ such that for any $s \in \xi(X, Y)$, the family is L-coercing for the map $F$ with respect to $s$.

Proof: Let us restrict to the case of $X=Z$ in Definition 2 and let $\left(C_{i}, K_{i}\right)_{i \in I}$ be the coercing family satisfying conditions of Definition 4 , since the set $I$ is nonempty, one can choose some $i_{0}$ in $I$.

By Condition (iii) of Definition 4, there exists some indice $k_{0}$ such that $\cap_{x \in C_{k_{0}}} F(x) \subset K_{i_{0}}$. We will denote by $K$ the set $K_{i_{0}}$, which is a compact subset of $X$. The set $C_{k_{0}}$ is contained in some compact convex subset $\tilde{C}$ of $X$. It is clear that

$$
\bigcap_{x \in \tilde{C}} F(x) \subset K \text {. }
$$


Let us define for all $a \in X, \tilde{C}_{a}=\tilde{C}$, we claim that the family (reduced to a single element) $\left\{\left(\tilde{C}_{a}, K\right)\right\}_{a \in X}$ is L-coercive for $F$ with respect to $s$. Indeed, condition $(i)$ is obvious, while condition (iii) follows from:

$$
\left\{y \in Y: y \in \bigcup_{z \in s^{-1}(y)} \bigcap_{x \in \tilde{C}_{z} \cap Z} F(x)\right\} \subset\left\{y \in Y: y \in \bigcap_{x \in \tilde{C}} F(x)\right\} \subset \bigcap_{x \in \tilde{C}} F(x) \subset K .
$$

Let us now consider $A \in\langle X\rangle$, the set $D^{A}=\operatorname{co}(\tilde{C} \cup A)$ is a compact convex subset of $X$ as convex hull of finite union of compact convex sets in a Hausdorff topological vector space (cf. Lemma 5.14 in $[\mathrm{AB}])$. It is clear that $A \subset D^{A}$ and that for all $x \in X, \tilde{C}_{x} \subset D^{A}$.

In the following example, we show that the converse of Proposition 1 is not generally true:

Example 1. For all $x \in \mathbb{R}$, we recall the usual notations $x^{+}=\max \{0, x\}$ and $x^{-}=\min \{0, x\}$. On the set $\mathbb{R}$, we will use the usual convexity as L-convexity and we put, for all finite subset $A$, $D^{A}=\operatorname{conv}(\{0\} \cup A)$.

Let us define the map $F: \mathbb{R} \rightarrow \mathbb{R}$ by $F(x)=\left[-x^{+}, x^{+}\right]$. The family $\left\{\left(C_{x}, K\right)\right\}_{x \in \mathbb{R}}$ defined, for all $x \in \mathbb{R}$, by $C_{x}=\left[x^{-}, x^{+}\right]$and $K$ is any compact set containing $\{0\}$ is L-coercing for $F$ with respect to the identity function. This family does not satisfy condition (ii) of Definition 4 since if we take $x>0$ and $y<0$, we cannot find $z \in \mathbb{R}$ such that $C_{y} \cup C_{x}=[y, 0] \cup[0, x] \subset C_{z}$.

The following example show that our coercivity is very general:

Example 2. Let $X$ be a convex subset of a Hausdorff topological vector space and $F: X \rightarrow X$ a map. Suppose that $F$ satisfies the following condition given in Theorem III of [L] or in Lemma 1 of [DT] (in term of the "dual" map): There is a nonempty compact convex subset $X_{0}$ of $X$ such that the set $\left\{y \in X: y \in \bigcap_{x \in \operatorname{conv}\left(X_{0} \cup\{y\}\right)} F(x)\right\}$ is compact.

Then, if we reduce $\Gamma$ to the usual convexity and $s$ to the identity map, the family $\left\{\left(C_{y}, K\right)\right\}_{y \in X}$ defined for all $y \in X$ by:

$$
C_{y}=\operatorname{conv}\left(X_{0} \cup\{y\}\right) \text { and } K=\left\{y \in X: y \in \bigcap_{x \in \operatorname{conv}\left(X_{0} \cup\{y\}\right)} F(x)\right\}
$$

is an L-coercing family for $F$ with respect to $s$ in the sense respectively of Definition 2 and Remark 3 by putting for each $A \in\langle X\rangle, D^{A}=\operatorname{conv}\left(X_{0} \cup A\right)$.

The following result is an extension of the lemma in [F1] to L-spaces. It also extends Theorem 000 in [L], Proposition 1 in [H2], Theorem 1 in [HL] and others ...

Lemma 1. Let $(X, \Gamma)$ be an L-space, $Z$ a nonempty subset of $X$ and $F: Z \rightarrow X$ a KKM map with quasi-compactly closed values. Suppose that for some $z \in Z, F(z)$ is quasi-compact, then $\bigcap_{x \in Z} F(x) \neq \emptyset$. 
Proof : The map $F$ have quasi-compactly closed values. Consequently, in view of the quasicompactness condition, it suffices to prove that for each finite subset $B$ of $X, \bigcap_{x \in B} F(x) \cap F(z) \neq \emptyset$, in order to prove that $\bigcap_{x \in Z} F(x) \neq \emptyset$. Let $B=\left\{x_{0}, \ldots, x_{n-1}\right\}$ be a finite arbitrary subset of $X$ and take $A=\left\{x_{0}, \ldots, x_{n}\right\}$, where $x_{n}=z$. Let $f: \Delta_{n} \rightarrow \Gamma(A)$ be the corresponding continuous function of $A$ satisfying for all $J \subset\{0, \ldots, n\}, f\left(\Delta_{J}\right) \subset \Gamma\left(\left\{x_{j} \mid j \in J\right\}\right)$. Then $\left\{f^{-1}\left(F\left(x_{i}\right) \cap f\left(\Delta_{n}\right)\right) \mid i=\right.$ $0, \ldots, n\}$ is a family of closed subsets of $\Delta_{n}$ such that $\Delta_{J} \subset \bigcup_{j \in J} f^{-1}\left(F\left(x_{j}\right) \cap f\left(\Delta_{n}\right)\right)$. It follows from Lemma in $[\mathrm{KKM}]$ that $\bigcap_{i=0}^{n} f^{-1}\left(F\left(x_{i}\right) \cap f\left(\Delta_{n}\right)\right) \neq \emptyset$, consequently $\bigcap_{x \in X} F(x) \neq \emptyset$.

Remark 5. The following example will show that the lemma can not be extended for compactly closed valued correspondence. Let $Z=X=[0,1]$ and $Y=X$ endowed with the trivial topology $\{\emptyset, X\}$. Let

$$
F(x)=\left\{\begin{array}{cll}
0, x] & \text { if } x>0 \\
\{0\} & \text { if } & x=0
\end{array}\right.
$$

It is easy to check that $F$ is KKM and that $F(0)$ is compact. Moreover, for this topology, any subset of $Y$ is compactly closed. Finally, it is obvious that the intersection is empty.

The main result of this paper is the following extension of the well known matching theorem of Fan (Theorem 4 in [F2]):

Theorem 1. Let $Z$ be an arbitrary set in the L-space $(X, \Gamma), Y$ an arbitrary topological space and $F: Z \rightarrow Y$ a map with quasi-compactly closed values. Suppose that there exists a function $s \in \zeta(X, Y)$ such that:

1. The map $R: Z \rightarrow X$ defined by $R(x)=s^{-1}(F(x))$ is $K K M$;

2. There exists an L-coercing family $\left\{\left(C_{x}, K\right)\right\}_{x \in X}$ for $F$ with respect to $s$;

Then $\bigcap_{x \in Z} F(x) \neq \emptyset$, more precisely $K \bigcap\left(\bigcap_{x \in Z} F(x)\right) \neq \emptyset$.

Proof: The map $F$ has compactly closed values. In order to prove that:

$$
K \bigcap\left(\bigcap_{x \in Z} F(x)\right) \neq \emptyset
$$

it suffices to prove that for each finite subset $A$ of $Z, \bigcap_{x \in A} F(x) \cap K \neq \emptyset$.

Let $A \in\langle Z\rangle$, by condition (ii) of Definition 2, there exists a quasi-compact L-convex set $D^{A}$ containing $A$ such that for all $y \in D^{A}, C_{y} \cap Z \subset D^{A} \cap Z$. Consider now the map $R^{A}: D^{A} \cap Z \rightarrow D^{A}$ defined by $R^{A}(x)=s_{0}^{-1}\left(F(x) \cap s\left(D^{A}\right)\right)$ where $s_{0}: D^{A} \rightarrow s\left(D^{A}\right)$ is the continuous function given by $s_{0}(x)=s(x)$ for any $x \in D^{A}$. By hypothesis (1) and the L-convexity of $D^{A}$, it is immediate that $R^{A}(x):=R(x) \cap D^{A}$ is a KKM map. Next, by the continuity of $s, F(x) \cap s\left(D^{A}\right)$ is closed in $s\left(D^{A}\right)$ then $R^{A}(x)$ is closed in $D^{A}$. Since $\left(D^{A}, \Gamma_{\mid<D^{A}>}\right)$ is also an L-space, we deduce by Lemma 1 that $\bigcap_{x \in D^{A} \cap Z} R^{A}(x) \neq \emptyset$. Since for all $x \in D^{A} \cap Z, s\left(R^{A}(x)\right) \subset F(x) \cap s\left(D^{A}\right)$, we have: 
$\bigcap_{x \in D^{A} \cap Z}\left\{F(x) \cap s\left(D^{A}\right)\right\} \neq \emptyset$. To finish the proof, we will show that: $\bigcap_{x \in D^{A} \cap Z}\left(F(x) \cap s\left(D^{A}\right)\right) \subset$
$\bigcap_{x \in A} F(x) \cap K$. Indeed, it is clear that $\bigcap_{x \in D^{A} \cap Z}\left(F(x) \cap s\left(D^{A}\right)\right) \subset \bigcap_{x \in A} F(x)$. Hence, it only remains to show that: $\bigcap_{x \in D^{A} \cap Z}\left\{F(x) \cap s\left(D^{A}\right)\right\} \subset K$. Let $y \in \bigcap_{x \in D^{A} \cap Z}\left(F(x) \cap s\left(D^{A}\right)\right)$, then $y \in s\left(D^{A}\right)$ which implies that there exists $z \in s^{-1}(y) \cap D^{A}$. By condition (ii) of Definition 2, $C_{z} \cap Z \subset D^{A} \cap Z$, and it follows that $y \in \bigcup_{z \in s^{-1}(y)} \bigcap_{x \in C_{z} \cap Z} F(x)$. Hence, by hypothesis (2), $y \in K$ and the theorem is proved.

Remark 6. Taking a continuous function $s$ in the previous theorem is inspired by Aubin ([A], p. 400), and has been used by [BC1], [BC2] and [L].

Now we can use Theorem 1 to prove the following result on fixed points :

Theorem 2. Let $(X, \Gamma)$ be an $L$-space, $Y$ an arbitrary topological space, $s \in \zeta(X, Y)$ and $S: X \rightarrow Y$ a map such that:

(i) For each $x \in X, S(x)$ is quasi-compactly open in $Y$;

(ii) For each $y \in Y, S^{-1}(y)$ is nonempty and L-convex;

(iii) There exists an L-coercing family $\left\{\left(C_{x}, K\right)\right\}_{x \in X}$ for the map $Q(x)=Y \backslash S(x)$ with respect to $s$.

Then, there exists $x_{0} \in X$ such that $s\left(x_{0}\right) \in S\left(x_{0}\right)$. In particular, if $s$ is equal to identity map, $S$ has a fixed point.

Proof: It follows by $(i)$ that $Q$ has quasi-compactly closed values and by (iii) that $\left\{\left(C_{x}, K\right)\right\}_{x \in X}$ is an L-coercing family for $Q$. Since for each $y \in Y, S^{-1}(y)$ is nonempty, then $\bigcap_{x \in X} Q(x)=\emptyset$. Now, let $R: X \rightarrow X$ be the map defined by $R(x)=s^{-1}(Q(x))$. We deduce by Theorem 1 that $R$ is not KKM. Let $A$ be a finite subset of $X$ and $x_{0} \in \Gamma(A)$ such that $x_{0} \notin \bigcup_{x \in A} R(x)$, it follows that $s\left(x_{0}\right) \in \bigcap_{x \in A} S(x)$. This means that $A \subset S^{-1}\left(s\left(x_{0}\right)\right)$, then by $(i i), \Gamma(A) \subset S^{-1}\left(s\left(x_{0}\right)\right)$. Hence $s\left(x_{0}\right) \in S\left(x_{0}\right)$.

Though [L] and [BC1], [BC2] use the notion of compactly-closed (without precising the compactness notion), in view of Remark 5, a cautious look at their papers shows that their assumption of compactly-closed corresponds (in our terminology) to quasi-compactly closed.

Note that Theorem 1 extends Theorem 1 in [BC1] and Theorem I in [L]. Theorem 2 generalizes Theorem 1 in [BC2] and Theorem $1.1 \mathrm{in}[\mathrm{L}]$. If the L-convexity is reduced to the usual convexity, then by Example 2, Theorem 1 and Theorem 2 extends respectively Theorem III in [L] and Lemma 1 in [DT]. Obviously, if $X$ is compact, the result of Theorem 1 follows immediately. 


\section{References}

[A] J.P. Aubin, Mathematical Methods of Game and Economic Theory, North-Holland, Amsterdam, 1979.

[AB] C. D. Aliprantis and K. C. Border, Infinite dimensional Analysis, Berlin, Springer, 1999.

[B] N. Bourbaki, General Topology: Elements of Mathematics, Chapters 1-4, (1989) Springer.

[BC1] C. Bardaro and R. Ceppitelli, Some further generalizations of Knaster-KuratowskiMazurkiewicz Theorem and Minimax Inequalities, J. Math. Anal. Appl. 132 (1989), 484-490.

[BC2] C. Bardaro and R. Ceppitelli, Fixed point theorems and vector valued minimax theorems, J. Math. Anal. Appl. 146 (1990), 363-373.

[BCFL] H. Ben-El-Mechaiekh, S. Chebbi, M. Florenzano and J-V. Llinares, Abstract convexity and fixed points, J. Math. Anal. Appl 222(1998), 138-150.

[BCF] H. Ben-El-Mechaiekh, S. Chebbi and M. Florenzano, A generalized KKMF principle, J. Math. Anal. Appl. 309 (2005), 583-590.

[C] S. Chebbi, Théorie du point-fixe dans le cas non-convexe et le cas non compact, Ph-D thesis, University Paris 1, (1997).

[DT] X.P. Ding and K.K. Tan, On equilibria of non compact generalized games, J. Math. Anal. Appl. 177 (1993), 226-238.

[F1] K. Fan, A generalization of Tychonoff's fixed point theorem, Math. Ann. 142 (1961), 305-310.

[F2] K. Fan, Some properties of convex sets related to fixed point theorems, Math. Ann. 266 (1984), 519-537.

[H1] C. Horvath, Some results on mutivalued mappings and inequalities without convexity, in "Nonlinear Analysis and Convex Analysis" (B. L. Lin and S. Simons Eds) (1987), 99-106, Dekker, New-York.

[H2] C. Horvath, Contractibility and generalized convexity, Journal of Mathematical Analysis and Applications, 156 (1991), 341-357.

[H3] C. Horvath, Extension and selection theorems in topological spaces with a generalized convex structure, Annales de la Faculté des Sciences de Toulouse, Vol. 2, nº 2 (1993), 253-269.

[HL] C. Horvath, and J.V. Llinares Ciscar Maximal elements and fixed points for binary relations on topological ordered spaces, Journal of Mathematical Economics, 25, vol. 3 (1996), 291-306.

$[\mathrm{KKM}]$ D. Knaster, C. Kuratowski and S. Mazurkiewicz, Ein Beweis des Fixpunktsatsez für ndimensionale simplexe, Fundamental Mathematics XIV (1929), 132-137.

[L] M. Lassonde (1983) : "On the Use of KKM correspondences in fixed point theory and related topics", Journal of Mathematical Analysis and Applications, 97, pp 151-201.

[PK] S. Park and H. Kim, Admissible classes of multifunctions on generalized convex spaces, Proc Coll. Natur. Sci. SNU 18 (1993), 1-21.

\section{S. CHEBBI}

King Saud University and Faculté des Sciences de Bizerte

Department of mathematics, P.O. Box 2455, Riyadh 11451, Saudia Arabia.

E-mail address: souhail.chebbi@laposte.net

P. GOURDEL

Paris School of Economics, University of Paris 1, CNRS,

CES, Université de Paris 1 Panthéon-Sorbonne, 106 Boulevard de l'Hôpital, 75647 Paris cedex 13, France. E-mail address: pascal.gourdel@univ-paris1.fr 
H. HAMMAMI

Ecole Polytechnique de Tunisie and Paris School of Economics, University of Paris 1, CNRS,

CES, Université de Paris 1 Panthéon-Sorbonne, 106 Boulevard de l'Hôpital, 75647 Paris cedex 13, France.

Ecole Polytechnique de Tunisie, B.P. 743, 2078 La Marsa, Tunis, Tunisia.

E-mail address: hakim.hammami@univ-paris1.fr 